\title{
The photosphere and veiling spectrum of T Tauri stars ${ }^{\star}$
}

\author{
H. C. Stempels and N. Piskunov
}

Department of Astronomy and Space Physics, Box 515, 75120 Uppsala, Sweden

Received 12 February 2003 / Accepted 22 April 2003

\begin{abstract}
Using recent high signal-to-noise and high spectral resolution $(R \sim 60000)$ observations with VLT/UVES, we have analysed the photosphere and veiling spectrum of five T Tauri stars. With a grid of 1-dimensional plane-parallel hydrostatic model atmospheres from the MARCS consortium we have determined their atmospheric properties, calculated synthetic spectra and determined the spectrum of the veiling continuum. Our analysis of the veiling spectrum supports the view that veiling can be represented by a combination of continuum sources. However, for the most strongly accreting stars we find a broad region around $5300 \AA$ where the derived level of veiling is consistently higher than expected from continuum sources.
\end{abstract}

Key words. stars: pre-main sequence - stars: atmospheres - accretion, accretion disks - methods: numerical

\section{Veiling in $T$ Tauri stars}

\subsection{CTTS and the accretion process}

Classical T Tauri Stars (CTTS) are a group of young, pre-main sequence stars of late spectral types. Typical characteristics of CTTS are strong emission lines, presence of $\mathrm{Li}$, weak photospheric absorption lines as well as strong variability in the continuum and emission line radiation. (see e.g. the review by Bertout 1989). These characteristics are commonly explained with the model of magnetospheric accretion (Ghosh \& Lamb 1979; Ushida \& Shibata 1985). In this model the stellar magnetic field couples to the circumstellar disk and controls the flow of matter from the disk to the star (Königl 1991). When this flow impacts on the stellar photosphere, shocks heat the flow of matter and the photosphere locally. In turn, hard X-ray radiation from these areas heats and ionizes matter in the accretion flow closest to the star. In the accretion shock region and the inner accretion region most of the observational characteristics of CTTS are formed, such as the strong emission lines. Weak-line T Tauri Stars (wTTS) are located in the same interstellar clouds as CTTS, contain Li and exhibit magnetic activity, but lack strong line emission and signatures of an accretion disk.

The model of magnetospheric accretion is supported by several observational diagnostics. High reddening indicates that these stars are often still surrounded by interstellar dust. Imaging and studies in the IR clearly support the presence of circumstellar disks. The observed magnetic fields on CTTS are relatively strong, up to $5 \mathrm{kG}$ or more

\footnotetext{
Send offprint requests to: H. C. Stempels, e-mail: Eric.Stempels@astro.uu.se

* Based on observations collected at the European Southern Observatory, Chile (proposal 65.I-0404).
}

(Johns-Krull \& Valenti 2000; Guenther et al. 1999), which is probably strong enough to completely dominate the flow of matter in the region closest to the star. Doppler Imaging studies of CTTS have revealed hot regions on the stellar surface (Rice \& Strassmeier 1996; Johns-Krull \& Hatzes 1997). Studies of the shapes of emission lines support the model of magnetospheric accretion (Muzerolle et al. 2001).

An excess of continuum radiation was first mentioned by Joy (1945) when he described T Tauri stars as a new class of stars. He observed "filling-in" of absorption lines, which reduces the effective line equivalent width. Studies of the overall flux distribution of CTTS have identified several continuous spectral components that do not have their origin in the stellar photosphere (Bertout et al. 1988). These components include excess IR radiation from the circumstellar disk, excess UV radiation from the accretion shock region, and b-f and f-f radiation from high-energy electrons in the inner accretion region. The particular feature we will focus on in this paper is the UV excess continuum.

\subsection{The veiling continuum}

The UV excess continuum appears as an additional continuum superimposed on the stellar photospheric spectrum. This effect is commonly called the veiling effect, and the excess continuum component the veiling continuum. Studies by Kenyon \& Hartmann (1987), Bertout et al. (1988), Basri \& Batalha (1990), Hartigan et al. (1991) showed that the veiling continuum covers a wide range of wavelengths, from far UV $(1000 \AA)$ all the way to the infrared ( $>10000 \AA$ ) and that it dominates the stellar flux shortward of the Balmer jump. The general spectral shape of the veiling continuum resembles a black body with a temperature in the range of 5000-20000 K, differing from star to star, with possible contributions from other continua such as 
the Paschen continuum. The relative size of the emitting region with respect to the stellar surface is usually expressed through the filling factor. Filling factors for TTS are typically $0.1-1.0 \%$ but sometimes as high as $10 \%$. As veiling is believed to originate from the accretion region it represents one of the most important diagnostics of the accretion flow in the vicinity of the stellar surface.

Because veiling closely relates to the accretion process, the level of veiling varies not only over wavelength, but also over time. In a previous study (Stempels \& Piskunov 2002, hereafter Paper I) we showed that it is possible to recover constraints on the temperature and size of accretion shock regions in RU Lup, one of the most active CTTS.

\subsection{Methods of measuring veiling}

Historically, measurements of veiling have been performed in two ways. These two methods have their basis in photometry and spectroscopy. With photometry one can easily recover the overall distribution of veiling by comparing absolute flux measurements over a long wavelength range with the predicted stellar flux spectrum. This method has been used by for example Bertout et al. (1988) to identify the different components in spectra of CTTS.

One can also determine veiling with spectroscopy, by comparing the line depths of photospheric absorption lines with a reference spectrum. This reference spectrum can be a synthetic spectrum or an observed spectrum of a star without veiling. This method, with wTTS and dwarfs as templates, was used in the instructive work of Hartigan et al. (1989) establishing an easy, reliable method for determining the veiling effect. The advantage of using spectroscopy is that one does not need flux measurements. However, the method based on spectroscopy requires high-resolution spectra with a relatively high signal-tonoise ratio (SNR) and adequate template spectra.

Valenti et al. (1993) measured the level of veiling using low-resolution spectra of broad vanadium and iron line complexes using wTTS as templates. In this way they were able to obtain veiling measurements for a few points in the optical range. They noted that their measurements are in agreement with high-resolution measurements of the same objects by Basri \& Batalha (1990).

A fourth method was proposed by Chelli (1998). He showed that it is possible to compare the shape of large-scale spectral features in low-resolution spectra of CTTS, such as the G-band, with spectra of reference stars, and in that way recover estimates of the veiling continuum. The work also showed that the photometric and spectroscopic methods yield more or less the same results.

\subsection{Goal of this paper}

In this paper we intend to demonstrate that the quality of atomic line data and model stellar atmospheres is now so good that it is possible to calculate synthetic template spectra for very accurate veiling measurements. Also, despite about 10 years of veiling measurements, it has not yet clearly been established what the sources are of the veiling continuum and whether there exist spectral features in the veiling spectrum. Using synthetic template spectra we separate the stellar photospheric spectrum from the spectrum of the accretion region in order to determine veiling spectra, as well as to verify the common assumption that the veiling continuum can be represented by a Planck function.

For CTTS in the northern hemisphere, veiling measurements over a range of wavelengths have been performed before (see Sect. 1.3), but either this range was relatively short, up to a few hundred $\AA$, or the obtained signal in the results was limited. The reason for this is that spectroscopic veiling measurements require a high spectral resolution and a high SNR in both the observed target and the observed reference spectrum, which is not easily combined with a long wavelength coverage.

The actual spectral shape of the veiling continuum requires special investigation, because it is still unclear how the energy released in the shock regions was processed before we detect it in our spectra. In order to address the above questions, we have performed a detailed analysis of high-quality spectra of a set of CTTS. In order to test the accuracy of our method of veiling determination we also determined veiling in two reference stars, the Sun and 61 Cyg A. In Sect. 2 we describe our set of observations, Sect. 3 addresses the veiling measurements and in Sects. 4 and 5 we present our conclusions.

\section{Observations}

During the nights of 16 and 17 April 2000 we obtained highresolution $(R \approx 60000)$ spectra for a number of CTTS with the UVES spectrograph on the ESO VLT/UT2 telescope at Paranal, Chile. The layout of the UVES spectrograph makes it possible to obtain simultaneously spectra through the blue and red arm of the spectrograph. This setup on such a large telescope makes it possible to obtain high-quality spectra throughout a large wavelength range, without compromising either the SNR, the spectral resolution or the duration of the individual exposures. Our spectra have typical exposure times of 15 to $30 \mathrm{~min}$ and a SNR of 100 for the blue and 200 the red wavelengths, or better. The wavelength coverage of our UVES setup is almost continuous from 3500 to $6700 \AA$. At two points in our observations the wavelength coverage is incomplete due to the transition from the blue to the red arm (4800-4900 $⿱$ ), and at the location of the boundary of the two CCDs in the red arm (near $5800 \AA$ ). However, it is clear that the performance of this instrument is well adapted to our specific requirements for an accurate study of the spectrum of the veiling continuum in CTTS.

Because possible spectral features may depend on the accretion rate or vary over time we observed time series of stars with accretion rates ranging from very strong to very weak. Because we also intend to investigate the short-term evolution of accretion and outflow diagnostics we restricted us to stars that are known to be photometrically variable and have documented changes in the strength of emission lines. During our run we observed RU Lup, CT Cha, CV Cha, RY Lup and Sz 68. We present an overview of these stars and their fundamental parameters in Table 1. 
Table 1. Observed targets and used model parameters. Data for the $V$-band magnitudes are from Herbig \& Bell (1988) and the SIMBAD database. Data for $v \sin i$ and $v_{\mathrm{rad}}$ and $\log \dot{M}$ for Sz 68 are from Johns-Krull \& Hatzes (1997); $\log \dot{M}$ for CT Cha is from Hartmann et al. (1989), for RU Lup from Lamzin et al. (2001) and for RY Lup from Gahm et al. (1993). No accretion rates were found in the literature for CV Cha. All other parameters (except for the Sun) are results of this paper. In addition to the tabulated values we used $[\mathrm{Fe} / \mathrm{H}]=0.0$ for synthetic spectra calculations.

\begin{tabular}{rrrrrrrrr}
\hline \hline Object & $\begin{array}{r}V \\
(\mathrm{mag})\end{array}$ & $\begin{array}{r}T_{\text {eff }} \\
(\mathrm{K})\end{array}$ & $\log g$ & $\begin{array}{r}v \sin i \\
\left(\mathrm{~km} \mathrm{~s}^{-1}\right)\end{array}$ & $\begin{array}{r}v_{\text {rad }} \\
\left(\mathrm{km} \mathrm{s}^{-1}\right)\end{array}$ & $\begin{array}{r}v_{\text {mic }} \\
\left(\mathrm{km} \mathrm{s}^{-1}\right)\end{array}$ & $\begin{array}{r}v_{\text {mac }} \\
\left(\mathrm{km} \mathrm{s}^{-1}\right)\end{array}$ & $\begin{array}{r}\log \dot{M} \\
\left(\log M_{\odot} \mathrm{yr}^{-1}\right)\end{array}$ \\
\hline CT Cha & $12.3-12.4$ & 4500 & 4.0 & 16.2 & 15.1 & 1.0 & 2.0 & -8.28 \\
CV Cha & $10.93-10.98$ & 4500 & 4.0 & 28.0 & 15.1 & 1.0 & 2.0 & - \\
Sz 68 & 10.4 & 4900 & 4.0 & 40.0 & -5.0 & 1.0 & 2.0 & $<-7.44$ \\
RU Lup & $6.9-13.4$ & 4000 & 4.0 & 9.0 & -1.9 & 1.0 & 2.0 & -6.52 \\
RY Lup & 11.1 & 5200 & 4.0 & 28.0 & -1.9 & 1.0 & 2.0 & $<-7.40$ \\
61 Cyg A & 5.21 & 4600 & 4.0 & 6.5 & -69.0 & 0.7 & 1.0 & \\
Sun & -26.7 & 5780 & 4.44 & 1.4 & -0.4 & 1.0 & 3.0 & \\
\hline
\end{tabular}

The data were reduced with the package REDUCE by Piskunov \& Valenti (2002). For a more detailed description of the reduction of this data set and for a comparison with spectra reduced with the UVES pipeline, we refer to Paper I.

\section{Measuring the veiling}

\subsection{Method}

We use the same method for calculating veiling as in our recent work on RU Lup. Therefore, we will only give a summary of the method used and the interested reader is referred to Paper I for more extensive derivations and details.

The contribution of the veiling continuum to the overall spectrum of CTTS is a function of wavelength. Therefore, it is appropriate to define the so-called veiling factor $V(\lambda)$, which is the relative strength of the veiling component compared with the stellar component at a certain wavelength $\lambda$. Here it is assumed that the veiling factor does not change throughout the observed interval around $\lambda$, which is true if the interval is short with respect to the typical size of the veiling continuum. Thus,

$V(\lambda)=I_{\mathrm{vc}} / I_{\mathrm{sc}}$

where $I_{\mathrm{vc}}$ is the intensity of the veiling continuum and $I_{\mathrm{sc}}$ the intensity of the stellar continuum. By defining $d$ as the unveiled relative line depth, and $d^{*}$ as the observed relative line depth, the veiling at a certain wavelength $\lambda$ can be determined by minimizing

$\chi^{2}=\sum\left\{\left(d^{*}-\frac{d}{1+V(\lambda)}\right) / \sigma_{d}\right\}^{2}$.

Here $\sigma_{d}$ is the standard error of the line depth $d$, which can be determined from the SNR of the observations in the continuum. The resulting formal error $\sigma_{V}$ of $V$ can be calculated from the standard relation $\sigma_{V}^{2}=2 / \nabla \chi^{2}(V)$, However, as will be shown later, the true error on the veiling measurements is larger than the formal error $\sigma_{V}$, because of small differences between the line shapes of synthetic spectra and observed spectra due to errors in the atomic data and limitations in our spectral synthesis calculations and stellar atmosphere models.

For this method to work, a template spectrum providing $d$ is needed. This can either be an observed non-veiled template or a synthetic spectrum. The method we use for veiling determinations is similar to the method used by Hartigan et al. (1989), with the only difference that in these studies observed spectra are used as templates for the underlying photosphere, while we calculate synthetic template spectra.

\subsection{Calculating synthetic spectra}

In recent years, major progress has been achieved in the development of lists of atomic line data and in calculations of stellar atmosphere models for late-type stars. Developments in these two fields are important for the calculation of accurate synthetic spectra, and, as we will show below, the quality of atomic line lists and model atmospheres is now so good that it is possible to calculate template spectra for veiling measurements in T Tauri stars.

We see several advantages in using synthetic templates instead of observed templates. The main advantage is that synthetic templates do not have to be observed and that for each target we can calculate which model atmosphere describes best the underlying photosphere, instead of having to use an observed template that is the closest spectral match with the target. Additional advantages that are especially important when measuring veiling are that synthetic template spectra are truly free from veiling and that radiative transfer calculations yield the level of the true continuum. Also, synthetic template spectra give us a better handle on the random errors of the observations and how these propagate into the final results.

The spectral line lists we used for our calculations are extracted from the publicly accessible Vienna Atomic Line Database (VALD, presented in Piskunov et al. 1995; Kupka et al. 1999; Ryabchikova et al. 1998). This database contains a large collection of line lists of atomic (and now also molecular) data. Also, for this database a number of data extraction tools were developed specifically for astrophysical purposes.

The development of model atmospheres for late type stars has taken a large step forward with the new generation of the MARCS (Gustafsson et al. 2003) and the Allard \& Hauschildt model atmospheres (Hauschildt et al. 1999). Currently, the quality of model atmospheres, as well as line lists is so good that the calculation of photospheric spectra for cool stars, such as the photospheres of T Tauri stars, is relatively 


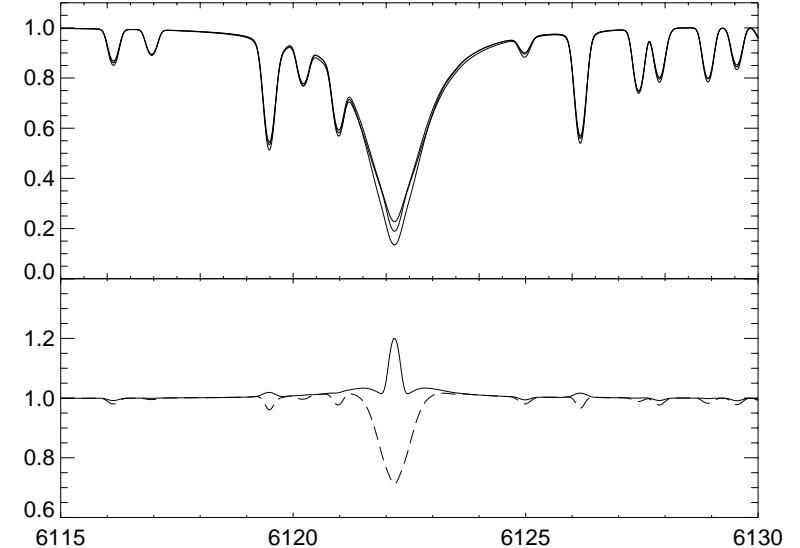

Fig. 1. A comparison of the MARCS ("M"), Kurucz ("K") and Allard \& Hauschildt ("A\&H") model atmospheres. Calculated line profiles of the Ca I $6122.2 \AA$ line are shown in the upper panel for the three models. From top to bottom these are the "M", "K" and "A\&H" models. In the lower panel we show the ratio of the calculated line profiles. The continuous line is ("M" / $\mathrm{K}$ ") and the dashed line ("A\&H" / $\mathrm{K}$ "). Units for the horizontal axis are $\AA$.

straightforward. Even when doing radiative transfer calculations with relatively simple assumptions such as plane-parallel, 1-dimensional model atmospheres in LTE and solar abundances, the results agree very well with observations (see also Sect. 3.5).

Today the MARCS models are perhaps the most complete 1-dimensional LTE models for cool stars in terms of line and continuous opacities as well as molecular equilibrium and opacity sampling calculations. We also produced synthetic spectra using the Allard \& Hauschildt and Kurucz models. We find an excellent agreement between all three models except in the cores of the strongest lines (see Fig. 1). This is consistent with the fact that the T- $\tau$-dependence generally is very similar for all three codes except for the outmost surface layers.

\subsection{Tuning the fundamental parameters of the targets}

In order to calculate synthetic template spectra for our target stars, the Sun and 61 Cyg A we first had to determine the fundamental parameters of these targets. These 7 fundamental parameters are $T_{\text {eff }}, \log g,[\mathrm{Fe} / \mathrm{H}], v \sin i, v_{\text {rad }}, v_{\text {mic }}$ and $v_{\text {mac }}$. During this process we concentrated on regions in our spectra that are well exposed and that contain lines with well-known atomic parameters covering a range of ionization potentials. For these selected regions, mainly in the red part of the spectrum, we extracted atomic line lists from the VALD database. In order to be able to simulate variations in the fundamental parameters we obtained a grid of solar abundance MARCS model atmospheres throughout the temperature range of interest (3500-5800 K) and for two values of $\log g$ (3.5 and 4.0).

Starting out from values for the fundamental parameters available in the literature, we then calculated for each region and star synthetic spectra with the SME package (Valenti \& Piskunov 1996). We then compared the calculated absorption line shapes with observed line profiles and adjusted the parameters if necessary. The major advantage of using the SME package is that it can do such a comparison and adjustment automatically through solving for the most likely values for the fundamental parameters by minimizing the differences between synthetic and observed profiles. As veiling may be present in the observations, we also solved for the level of veiling. However, during the tuning of the fundamental parameters the level of veiling was only treated as an additional free parameter, and was not yet intended as a measurement.

From our analysis with SME we could not detect systematic differences between the observed and synthetic spectra of both shallow and deep lines, which could be indicative of non-solar abundances; therefore we assumed solar abundances $([\mathrm{Fe} / \mathrm{H}]=0)$ for all targets. This conclusion is supported by Padgett (1996), who observed and analyzed spectra of T Tauri stars from several nearby star formation regions and found no direct indication that abundances are considerably non-solar. For CV Cha and RY Lup, stars that are in both her and our sample, she found respectively $[\mathrm{Fe} / \mathrm{H}]=-0.09 \pm 0.11$ and $0.09 \pm 0.13$.

The effect of $v \sin i$ and $v_{\text {rad }}$ on the shape of spectral lines is so strong that it is relatively straightforward to solve for these parameters. In the case of $T_{\text {eff }}$ and $\log g$ we made SME perform a search through our grid of model atmospheres. Finally, $v_{\text {mic }}$ and $v_{\mathrm{mac}}$, the parameters that line profiles are least sensitive to, were tuned to reflect the best correspondence with the observed profiles while maintaining physically acceptable values. For all stars (except when comparing with the Sun and 61 Cyg A, see below) we adopted the values of $v_{\text {mic }}=1.0$ and $v_{\text {mac }}=2.0$.

The final fundamental parameters we obtained for our program stars for producing the final synthetic spectra are listed in Table 1 .

\subsection{Measurements}

Having determined the fundamental parameters of our program stars we finally could measure veiling in out targets with the method described in Sect. 3.1. In order to measure the veiling factor at a range of wavelengths we extracted for each target at approximately every $100 \AA$ a segment of about $50 \AA$ throughout the entire reasonably well observed wavelength range (3900-6700). Again, we extracted for each region atomic line data from the VALD database for these segments. One single line list was used for all calculations.

As spectra of T Tauri stars contain a large number of emission lines and there are absorption lines present in the observed spectra that are not identified in our line lists, it is not possible to use all points of each observed segment for our veiling measurements. Also, because the line depth $d$ is measured relative to the continuum level in the spectrum, such continuum regions need to be identified. Therefore, in order to reject regions with inaccurately modelled profiles and to accurately determine the line depth $d$ and continuum SNR of each segment, we manually selected for each star and spectral segment (1) regions that are contaminated with emission lines or that contain unidentified spectral features, and (2) regions of the stellar continuum.

In Figs. 2 to 8 we show representative samples of comparisons between the artificially veiled synthetic template and the observed spectra. The obtained residual corresponds to what 


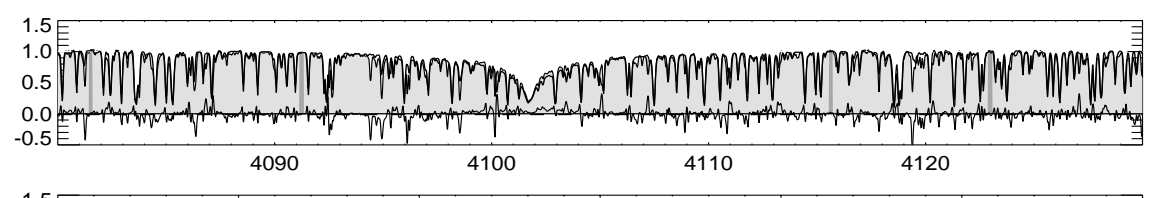
4355
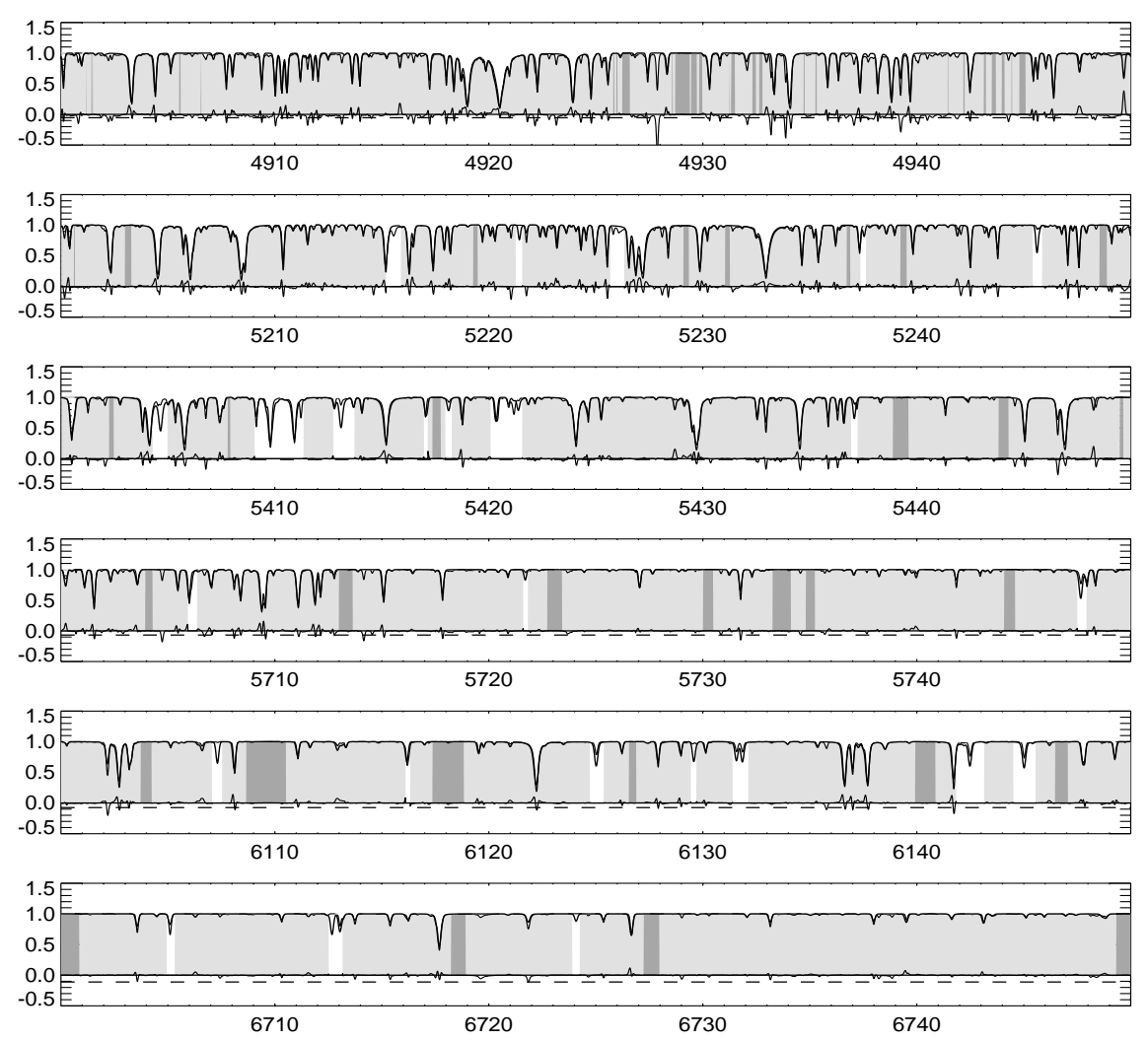

Fig. 2. A selection of representative spectral regions that were used to determine the veiling factor in the Sun. We plot the observations (thin line), the veiled synthetic spectrum (thick line) and the contribution of the veiling continuum (dashed line). The residual of the observations and the veiled synthetic spectrum is also shown (thin line near the horizontal axis).

The dark grey areas were used to establish the continuum level of the observations and the white areas were excluded when determining the veiling factor. Units for the horizontal axis are $\AA$.

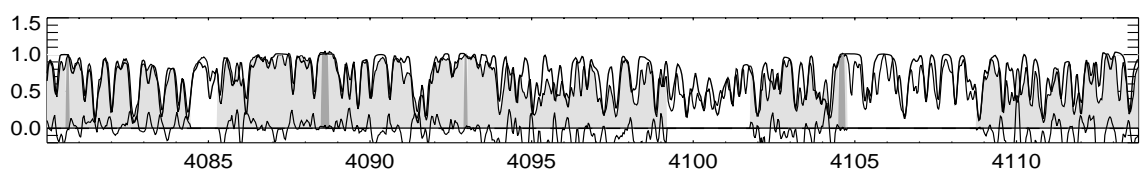

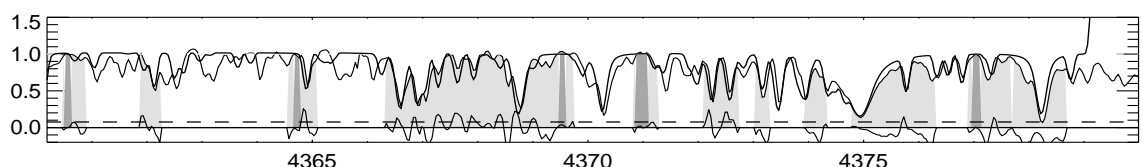
1936 5205 $\frac{5410}{5420}$ 0.0 (150 1.5

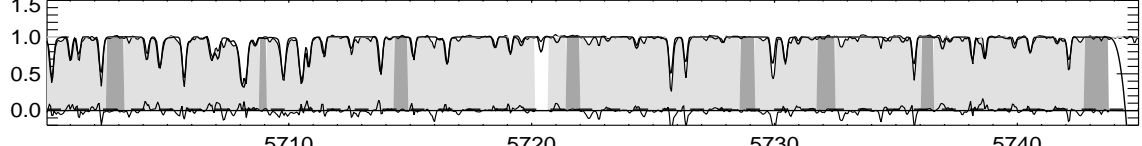
0.0 (10

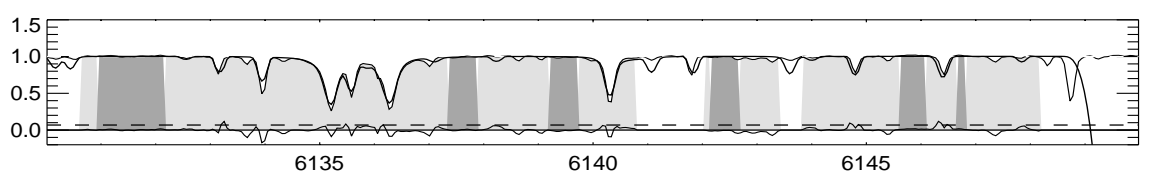

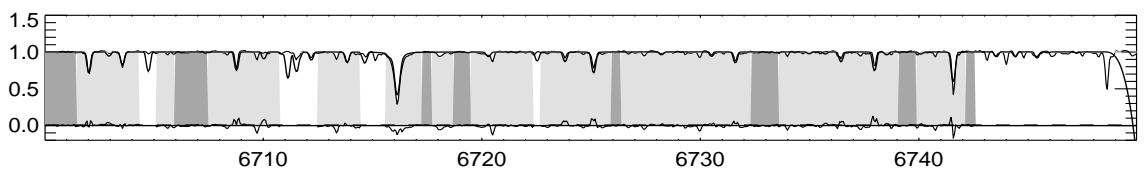

Fig. 3. Same as Fig. 2, but for 61 Cyg A. Note that for this star the selection of wavelength regions is slightly different (see Sect. 3.5). 


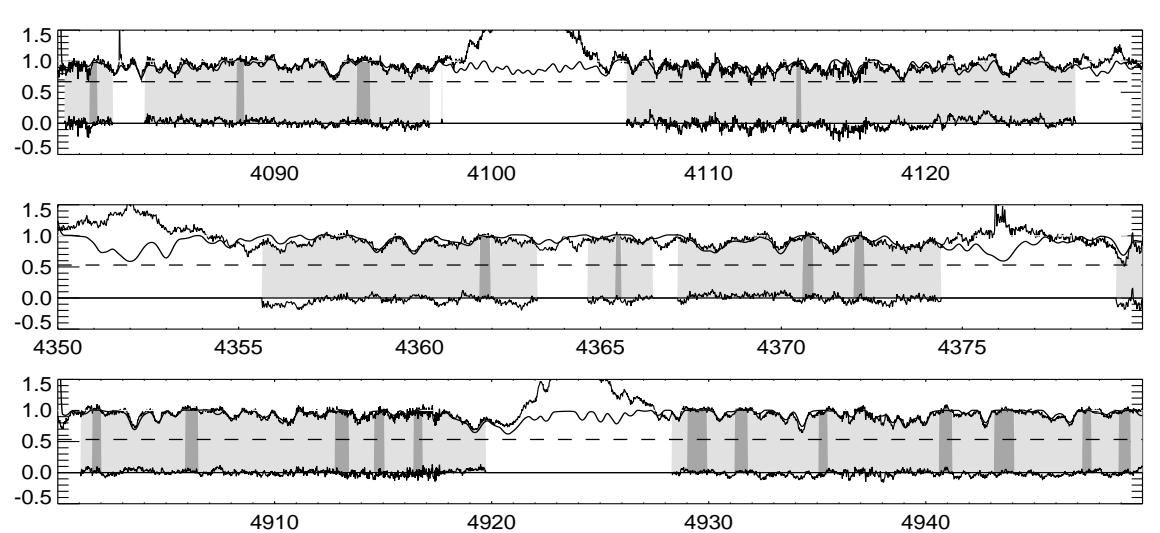

(210

1.0 .5

0.0 (1)

$5410 \quad 5420 \quad 5430 \quad 5440$

-0.5

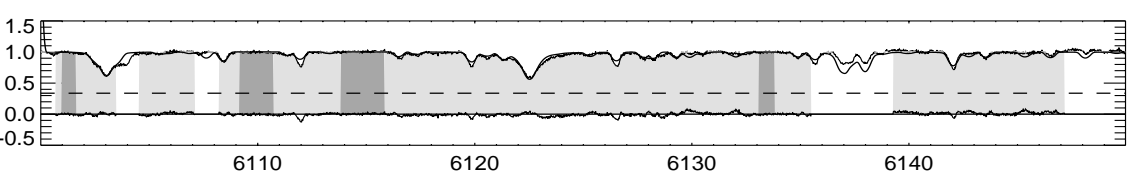

$\int_{6710}^{1.0} \int_{-0.5}^{1.5}$

Fig. 4. Same as Fig. 2, but for CT Cha.

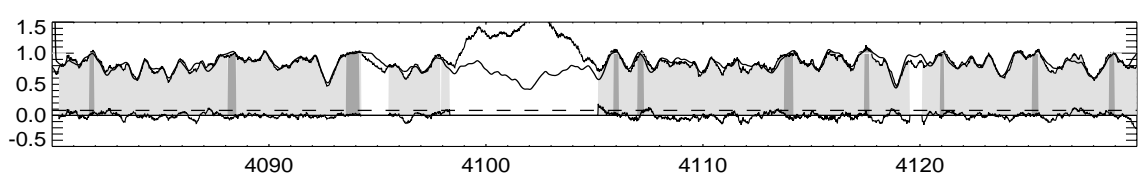

0.0)

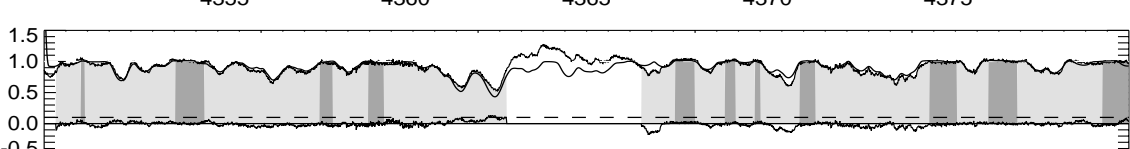

0.0 (0.5)

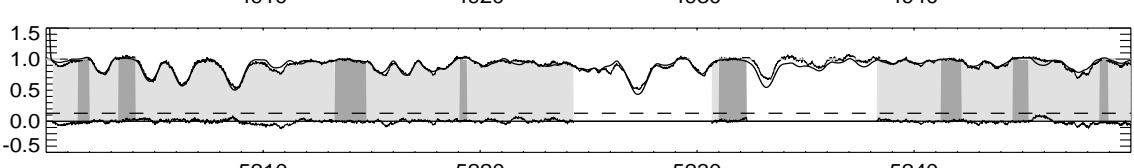

5210

5220

5240

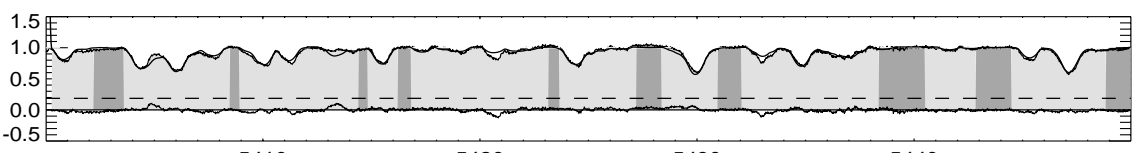

5410

5430

5440
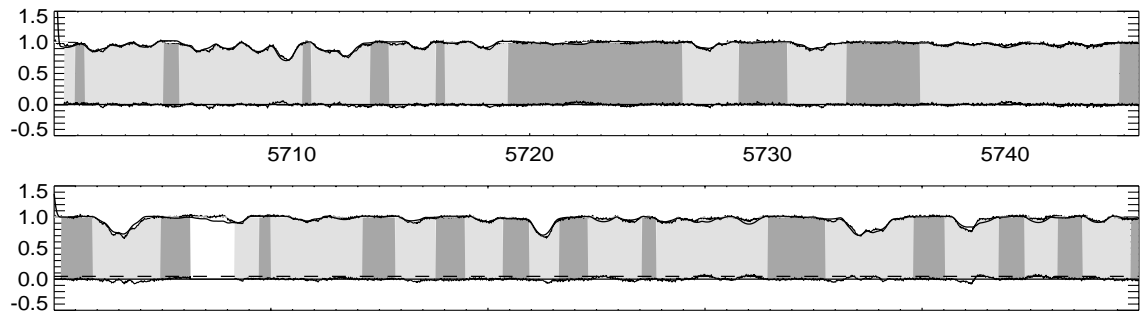

6110

6120

6140

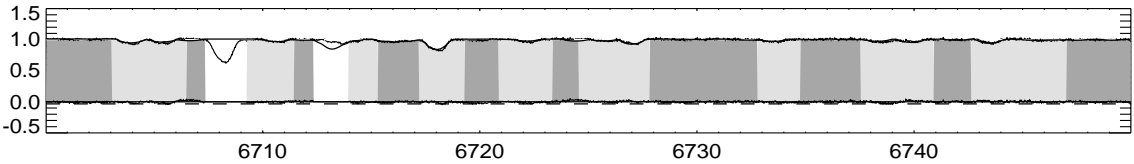

Fig. 5. Same as Fig. 2, but for CV Cha 

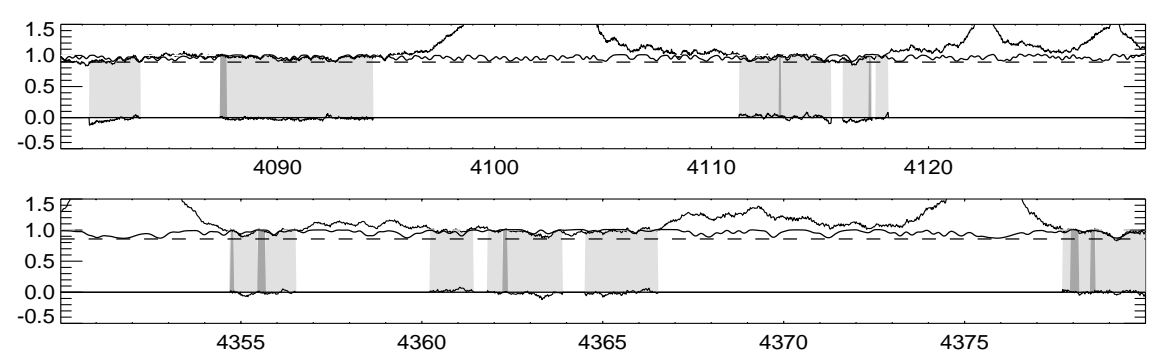

$$
\begin{array}{rlllll}
4355 & 4360 & 4365 & 4370 & 4375
\end{array}
$$

0.5

$$
\begin{array}{lllll}
4900 & 4910 & 4920 & 4930 & 4940 \\
\hline
\end{array}
$$$$
0.0
$$$$
\begin{array}{r}
0.0 \\
-0.5 \\
\hline
\end{array}
$$$$
\begin{array}{rrrr}
5210 & 5220 & 5230 & 5240
\end{array}
$$$$
\text { (1.0) }
$$

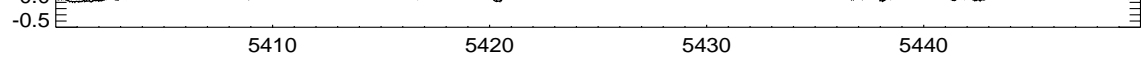$$
\text { (1.0) }
$$

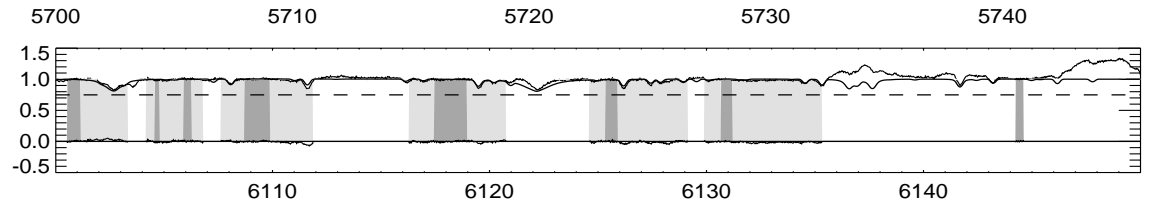

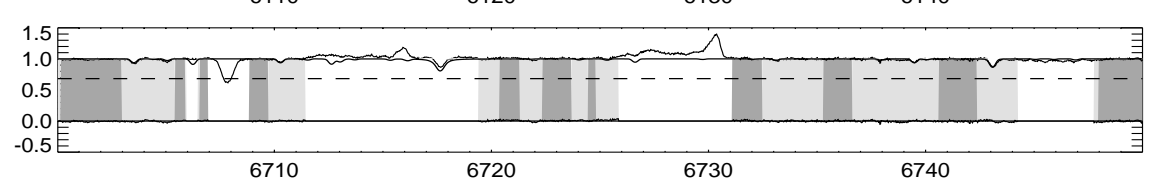

Fig. 6. Same as Fig. 2, but for RU Lup. $\underbrace{1.5}_{4090}$ ${ }_{4355}$

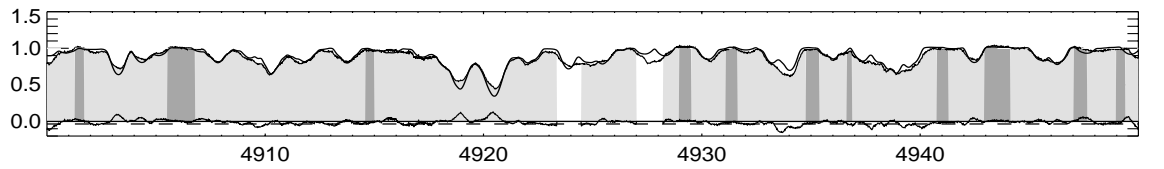
\begin{tabular}{llll}
\hline 4910 & 4920 & 4930 & 4940
\end{tabular}

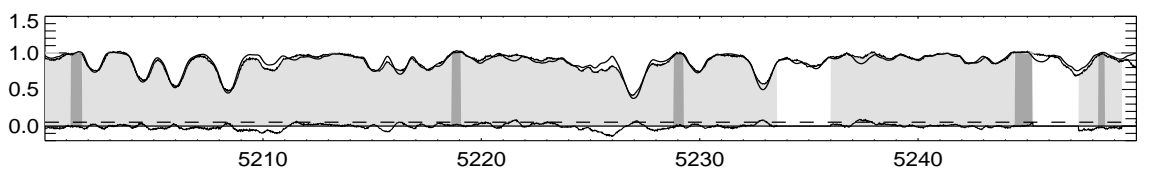
5210 5220 5230 524

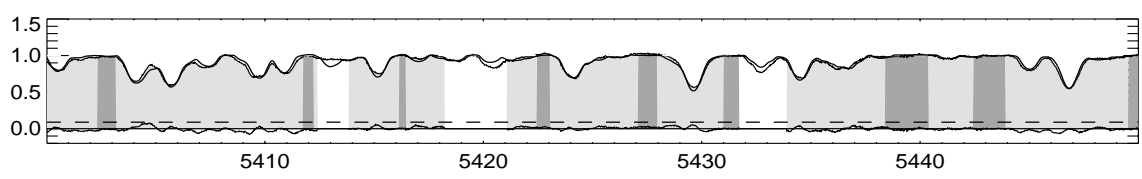
5410 5430 5440
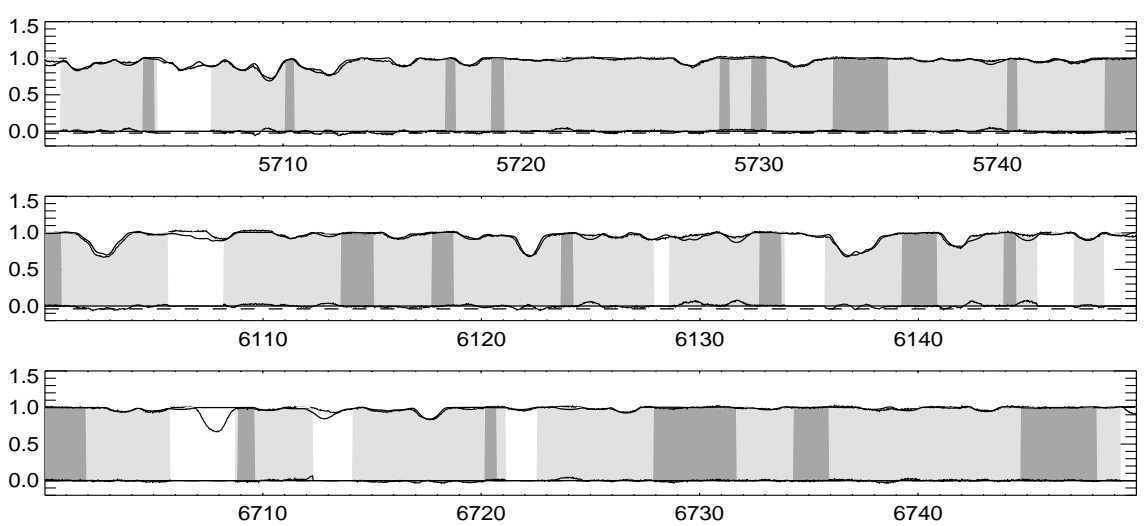

Fig. 7. Same as Fig. 2, but for RY Lup. 


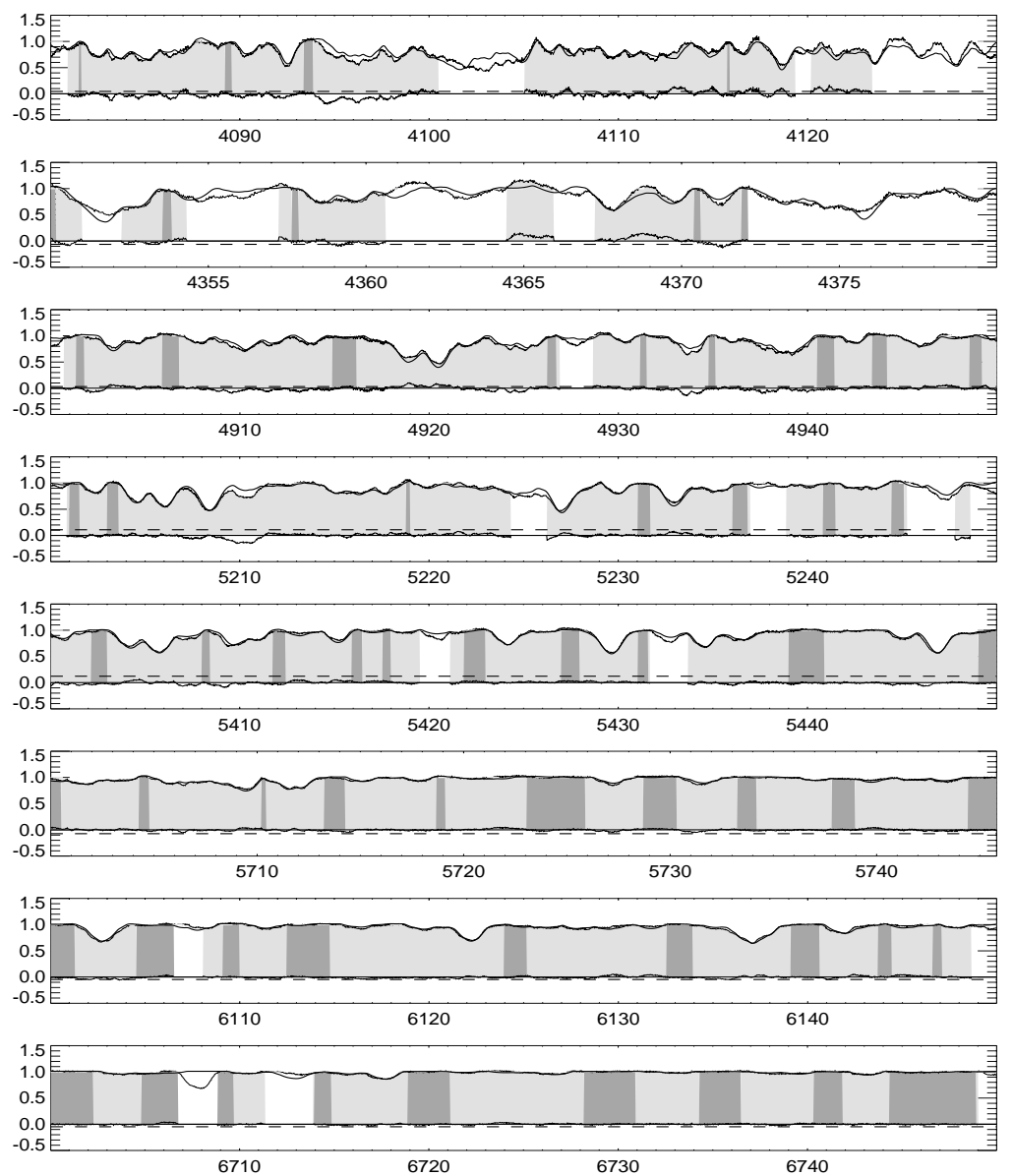

Fig. 8. Same as Fig. 2, but for Sz 68.

can be expected from the SNR of the observations. The fact that we could properly reproduce the shape of hundreds of photospheric lines with equivalent widths ranging from a few $\mathrm{m} \AA$ to several hundred $\mathrm{m} \AA$ supports that plane-parallel hydrostatic model atmospheres as well as LTE spectra synthesis are adequate for obtaining good veiling measurements.

The distribution of the veiling factors we obtained in this way are presented in the upper panels of Fig. 9. Veiling values themselves are good indicators for the relative contribution of non-photospheric sources, but have little physical value. Therefore, in order to study the physical processes that cause the veiling effect, we converted veiling factors to veiling fluxes by multiplying them with the theoretical continuum fluxes of the atmospheric models that were used in the synthetic template spectra calculations. The veiling flux spectra are presented in the lower panels of Fig. 9. We also include the model atmosphere continuum flux for comparison.

\subsection{Comparison with reference stars}

In order to assess the accuracy of our method of veiling determination, we performed our veiling analysis on two reference stars, the Sun (spectral class G5V) and the 61 Cyg A (K5V similar to our CTTS targets). For these main-sequence stars we do not expect to recover any veiling. We performed our synthetic spectra calculations in exactly the same way as for the other objects. We would like to emphasize that we did not tune the atomic line list nor the model atmosphere for these stars. We list our choice of fundamental parameters for the Sun and 61 Cyg A in Table 1.

The observed solar spectrum we used for veiling determination was taken from the NSO solar atlas (Kurucz et al. 1984). We did not degrade the spectral resolution or SNR of the solar spectrum because we intend to obtain an estimate of the accuracy of our method. The spectrum of $61 \mathrm{Cyg}$ A originates from the 2D-Coudé echelle spectrometer at McDonald Observatory. It has a spectral resolution of 60000 and a SNR of more than 300 , which is comparable to our CTTS data. However, the wavelength coverage of this star is not continuous as in our UVES data. Because we want to obtain estimates of the accuracy of the veiling determination in the regions we selected for our CTTSs, and in particular the atomic line lists, we only investigated $61 \mathrm{Cyg} \mathrm{A}$ in those spectral regions that overlap with the regions selected for our CTTS.

Similar to the method we applied to our other objects we constructed a mask of regions that indicate unidentified spectral features, as well as regions of the stellar continuum, after which we solved for the veiling factor. Meticulously comparing the observed spectra with the final synthetic spectra, we conclude the following:

- We find that on average the recovered veiling factor in the Sun is slightly negative $(-0.05)$. This offset from the expected value $(0.00)$ can be traced to line asymmetry in the 

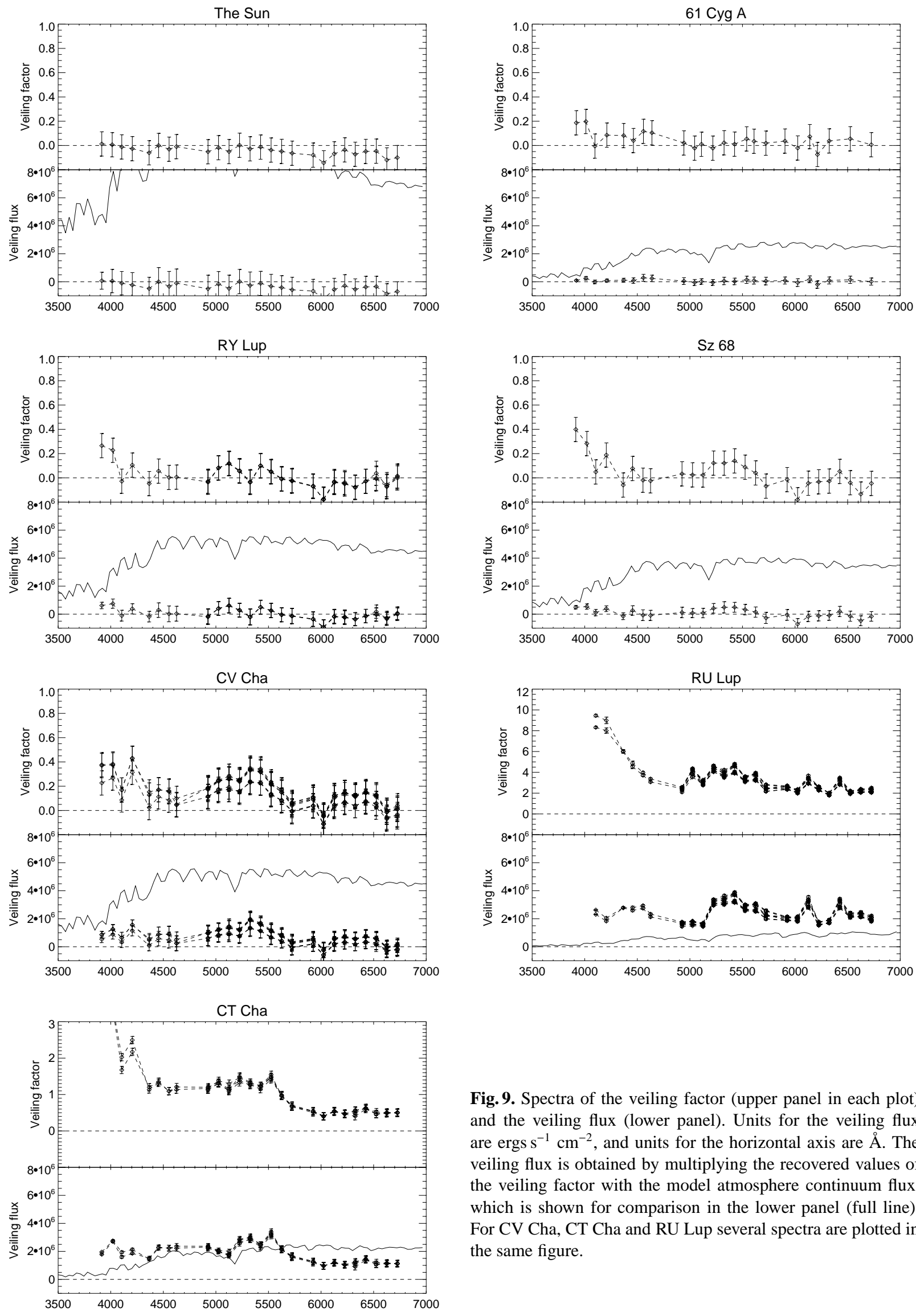

Fig. 9. Spectra of the veiling factor (upper panel in each plot) and the veiling flux (lower panel). Units for the veiling flux are ergs s$~^{-1} \mathrm{~cm}^{-2}$, and units for the horizontal axis are $\AA$. The veiling flux is obtained by multiplying the recovered values of the veiling factor with the model atmosphere continuum flux, which is shown for comparison in the lower panel (full line). For CV Cha, CT Cha and RU Lup several spectra are plotted in the same figure. 
solar spectrum which is not reproduced by our LTE hydrostatic calculations and models.

- The veiling values in $61 \mathrm{Cyg} \mathrm{A}$ are all close to the expected value $(0.00)$, except in the bluest part of the spectrum. For this star the line shapes are dominated by the instrumental profile and therefore any line asymmetry will not affect our veiling measurements. For cool late-type stars there are hardly any continuum points in these regions, making veiling estimates difficult. This implies that veiling values in the blue may be slightly overestimated.

- The 1- $\sigma$ random errors (scatter) on our measurements of the veiling factor are about 0.1 . This is due to the fact that inaccuracies in the veiling determinations are dominated by inconsistencies between the calculated line profiles and the actual observed line profiles. Such inconsistencies are mainly due to small errors, incompleteness in our atomic data and NLTE effects. However, this limitation does not lead to excessive random errors nor to systematic errors in the veiling determinations.

The formal error of the veiling measurements, $\sigma_{V}=$ $\left(2 / \nabla \chi^{2}(V)\right)^{\frac{1}{2}}$, is of the order of 0.01 , which shows that the empirically determined errors exceed the formal errors. It is important to realize that the Sun with its very low $v \sin i$ and the near-to-infinite SNR of the FTS spectra represents a rather extreme case. In the particular case of the Sun small errors and missing lines in our line list are likely to give a more pronounced effect on our measurements compared to the other targets. For observations at a lower spectral resolution and for stars with larger $v \sin i$ the effect on the line depth, and thus also on the recovered veiling values, due to NLTE effects or incompleteness of the line list, is reduced. Therefore, we expect the empirically determined error for the Sun to be an upper limit for the other targets. However, at large veiling values the formal error starts dominating, and the error margin should be adjusted upwards. Thus we choose the error $\sigma_{V}$ we quote in our veiling determinations to be the larger of 0.1 or $\left(2 / \nabla \chi^{2}(V)\right)^{\frac{1}{2}}$. We do not expect that an offset in the average veiling factor due to subtle effects in the upper atmosphere can be observed in targets with larger values for $v \sin i$. Even if it were detectable, a systematic offset to our veiling measurements will not influence our conclusions on the spectral shape of veiling.

Even though our choice of our error margins (typically 0.1 ) on the veiling factor is rather conservative, the error margins obtained with synthetic templates from our high-SNR spectra are better than the error margins from previous studies with "conventional" methods using non-veiled template stars. For example, the random errors on the veiling measurements of Hartigan et al. (1991), the most detailed study of its kind, are typically 0.2 for the brightest stars.

From the comparison of the synthetic spectrum with the spectra of the Sun and 61 Cyg A we can also see that our line list is almost complete for the wavelength range 5000-6700 $\AA$, which is also the region where the observations of our targets have the best SNR.

\section{Discussion}

Although the level of the veiling flux is as expected quite different for different objects, the obtained spectra show some resemblance to each other at least for the most active stars. The two most obvious properties are the rise toward the blue and a rather flat shape in the red.

Contrary to what one might expect, there is no strong correlation between the veiling intensity and accretion rates from the literature. This is mostly apparent for CT Cha, for which the level of veiling is moderate to strong while the published accretion rate is relatively low. This may be explained by the fact that accretion rates from the literature represent a different epoch than our observations. Also, accretion rates are often measured using processes that may represent other formation mechanisms than the optical excess flux that is measured in our method. For example, Lamzin et al. (2001) determined the accretion rate of RU Lup from the intensity of Fe coronal lines, while Johns-Krull \& Hatzes (1997) and Hartmann et al. (1989) determine the accretion rates of respectively Sz 68 and CT Cha from the excess luminosity due to heating of freely infalling material. Gahm et al. (1993) infer the accretion rate of RY Lup from the infrared excess luminosity and the corresponding color changes during luminosity variations.

\subsection{The spectrum of the veiling continuum}

For RY Lup and Sz 68 the veiling spectrum is very weak and flat, and it is not possible to derive any conclusions about the source of the veiling flux, if any. The shape of the bluest part of the veiling spectrum is similar to shape obtained for $61 \mathrm{Cyg}$ A, and we consider this an artifact. The absence of veiling in these stars confirms that our choice of solar abundances was correct. The flux spectra of CT Cha and RU Lup are clearly visible and similar in shape. The intermediately active star CV Cha shows a low level of veiling and the shape of the veiling spectrum shows some of the characteristics that are seen in the spectra of CT Cha and RU Lup.

The overall shape of the veiling spectra we recovered for the most strongly accreting stars (CT Cha and RU Lup) is dominated by a continuous component. Towards the bluest wavelengths the contribution of the veiling spectrum is largest, which is consistent with the idea that veiling is produced by continuous emission from hot plasma flows around the star.

However, in the wavelength range 5000-5700 $\AA$ we find for all actively accreting stars that the recovered intensities of the veiling spectra of CT Cha and RU Lup, and in a less pronounced fashion, in $\mathrm{CV}$ Cha and $\mathrm{Sz} 68$, have higher values that what is expected from the continuous UV excess continuum. In fact, such a narrow feature cannot be reproduced by any single or combination of stellar continua with different temperatures, because of the large range of wavelengths that Planck functions affect. The shape of the feature is also incompatible with other continuum sources of the veiling spectrum, such as the Paschen continuum. This feature is absent in the Sun and 61 Cyg A, and well outside our error bars. This is an unexpected result, since non-continuous features in the veiling spectrum have not been 

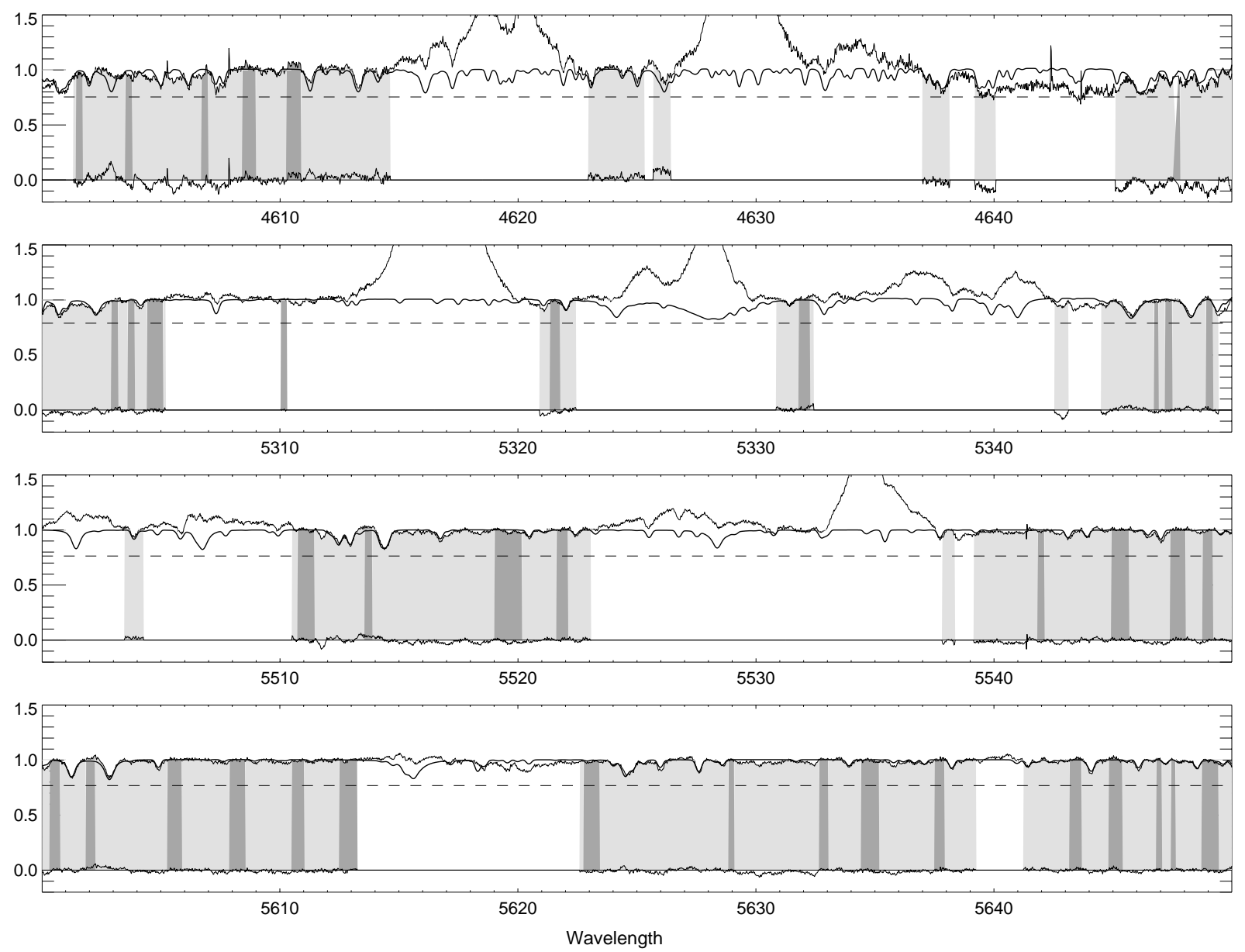

Fig. 10. This plot shows in detail the quality of the obtained solutions for the level of veiling in RU Lup. These panels show the regions in the spectrum of RU Lup that were not included in Fig. 6. The high values of veiling recovered in the region 5000-5500 (see Fig. 9) are not due to misinterpretation of strong line emission. Large regions without strong line emission are present in all panels. A high value for the veiling factor is also recovered for the region 5600-5650 which is void of emission lines.

reported before. However, if such emission regions exist in the veiling flux this opens a new window to the accretion regions.

In order to investigate that this region of enhanced veiling is a real feature we considered three possible origins of systematic errors: (1) instrumental artifacts, (2) systematic errors in the method of veiling measurements and (3) enhanced contribution of line emission that is misinterpreted as a veiling continuum.

The first alternative is easily dismissed because of consistency of the observations. Any systematic errors due to instrumental artifacts can be excluded, because the region of excess veiling only appears in the veiled targets, even though all five CTTS targets were observed with an identical instrument setup and identical calibration procedures.

The second alternative, systematic errors in the method of veiling measurements, can be excluded because we found zero veiling for the Sun, for 61 Cyg A as well as for the least active star RY Lup. We also investigated what the effect is of restricting our veiling measurements to deep or shallow lines, because in active stars such as wTTS "filling-in" of the core of strong absorption lines occurs. We only found small differences in veiling that did not exceed the error bars of our measurements. In addition, we know from spectral synthesis what the shape and depth of unperturbed strong and shallow absorption lines is, which means that we can identify deep absorption lines with profiles that are considerably different from the synthetic spectra (for example the Mg lines near $5120 \AA$ in RU Lup).

In order to test the third alternative, that the region of enhanced veiling is actually the result of line emission, we carefully examined our veiling measurements for all segments in the region 5000-5700 $\AA$ looking for a systematic overestimate of the measured veiling values due to the presence of emission lines. However, we could not find any relation between the intensity of emission lines and the measured veiling value. This is illustrated in Fig. 10, in which we show large-scale plots of the spectral regions that were not included from Fig. 6. For example, there are spectral regions with high veiling values that contain relatively few emission lines (around $5600 \AA$ ), as well as spectral regions with concentrations of strong emission lines, but relatively low veiling values (around $4500 \AA$ ). Also, we do not find any correlation between the derived veiling values and the strength of the emission lines in any given interval.

We also discard the possibility that the excess veiling values can be caused by the presence of many unidentified weak emission lines. The residual differences between veiled synthetic spectra and CTTS observations with residuals for the solar spectrum fits are, taking into account the difference in 


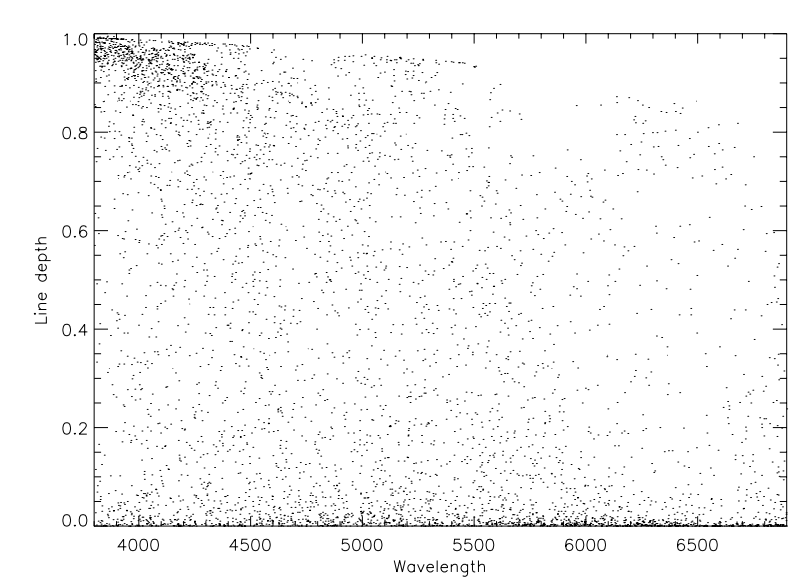

Fig. 11. This figure shows the distribution of the line depth versus wavelength of Fe lines that are present in the VALD database. The line depths were calculated for $T_{\text {eff }} 4000 \mathrm{~K}$ and $\log g=4$. Within the observerd wavelength range, the ratio of strong and weak lines changes very slowly. Therefore, we do not expect emission from an ensemble of weak lines to be able to cause excess veiling values in the range 5500-5700 ̊ where no strong line emission is seen.

signal-to-noise ratio, of similar magnitude. This is not surprising because the absorption spectrum consists mostly of lines of neutral species, while the emission line spectrum consists of singly ionized metals. In the case of iron lines, we know from statistics based on data in the VALD database that in our spectral range the wavelength distribution of weak and strong lines is very similar (Fig. 11). Therefore, the equivalent width of strong emission lines in every interval can be used as a proxy for a "haze" of undetected emission which could be mistaken for veiling. As we find no correlation between veiling and strong line emission, we conclude that any effects due to the third alternative are insignificant.

One can also reject the contribution of coronal emission lines. As is shown by Lamzin et al. (2001) the contribution of coronal emission lines in the optical part of the spectrum is far beyond our current detection limits.

\subsection{Comparison with other studies}

Although regions of enhanced veiling have not been reported before, there is some indication that such regions are visible in other investigations. Hartigan et al. (1991) measured veiling for 22 CTTS and wTTS in Taurus and Auriga over the range $4000-6800 \AA$. Their results show that veiling values can range from as little as 0.1 to about 10 , and they conclude that the wavelength distribution of veiling is like that of a continuum. Also in their veiling measurements of actively accreting CTTS, one can see there is a region with enhanced veiling measurements around $5500 \AA$ but it often disappears in the scatter of their measurements. The fact that they recover similar results using stellar templates indicates that the region of enhanced veiling is not caused by our synthetic templates. However, it does indicate that this region only appears in CTTS and not in wTTS.
Basri \& Batalha (1990) published veiling spectra for 35 TTS using Hyades dwarfs as templates. In their spectra, there is no region with enhanced veiling measurements present. The scatter in their plots is large, but in some plots the feature should have been visible.

Calvet \& Gullbring (1998) investigated the shape of the veiling continuum over a very large wavelength range (200-20000 Å). They modelled radiation from a K7-M0 photosphere with and without an accretion column, and determined the ratio. Their model shows that for typical surface filling factors of a few percent the veiling factor strongly increases towards the bluest wavelengths, while towards the redmost wavelengths the veiling factor becomes more or less constant. This is in quantitative agreement with our observations of RU Lup, CT Cha and CV Cha, but does not explain the region of enhanced veiling around $5000-5700 \AA$.

The only star in out sample for which veiling measurements were performed before is Sz 68. Johns-Krull \& Hatzes (1997) measured veiling for 7 spectral lines in the interval 6190-6707 $\AA$ and found a mean veiling factor of $0.05 \pm 0.06$, which agrees with the values we obtained in this study.

\subsection{Evolution of veiling}

For three of the stars studied in this paper, CT Cha, CV Cha and RU Lup, we obtained more than one spectrum (see Fig. 9). The time evolution of spectra of CTTS deserves to be investigated in detail, because of the close relationship between veiling and the accretion process. In Paper I we analyzed our observations of RU Lup, and showed that the changes of the veiling factor on short time scales can be interpreted as variations in the amount of material accreted and the visible area of the accretion region on the star.

The variations in the level of veiling of CT Cha and CV Cha is much less pronounced that what we find for RU Lup (Paper I). Variations consist mostly of relatively small local changes in veiling, while the overall shape of the veiling spectrum is only affected on much longer time scales. The smallscale changes are small in comparison to the expected statistical variation, and their behavior is not unlike the changes in emission line shapes (see Figs. 8 to 11 in Paper I).

\subsection{Effective temperatures and magnetic fields}

That magnetic fields as strong as $1-2 \mathrm{kG}$ are present around CTTS is generally accepted (Johns-Krull \& Valenti 2000; Guenther et al. 1999), but the actual geometric configuration and the interaction with the disk are still open problems. However, without addressing these questions directly it is possible to predict some of the consequences of the presence of magnetic fields in CTTS on the resulting veiling spectrum.

First of all, in the picture of magnetospheric accretion the magnetic fields play a major role in channelling the accretion of material from the circumstellar disk to the stellar surface. This flow of matter will give rise to hot accretion regions in the photosphere. Also the presence of strong magnetic fields will cause cool spots to appear in the non-accreting regions of the 
stellar surface. The presence of such hot and cool areas may influence the value of the veiling factor. While the continuous veiling component is considered to originate from hot regions on the stellar surface, the consequences of the presence of cool regions, though expected, is not as clear. Such cool spots will influence photometric measurements and also change the apparent effective temperature.

The latter effect is relevant for our calculations, because we use one single $T_{\text {eff }}$ for our synthetic spectra calculations. Cool regions on the surface will cause an underestimate of the stellar continuum flux and thus an overestimate of the veiling factor. However, this effect is small even if the cool spots cover a moderate to large area of the stellar surface. For example, a star that is for $50 \%$ covered with areas of $T_{\text {eff }}$ of $5000 \mathrm{~K}$ and for $50 \%$ with areas of $3800 \mathrm{~K}$ will produce a total flux equivalent to a star with $T_{\text {eff }}=4500 \mathrm{~K}$. Using our method of veiling determination with synthetic spectra for $T_{\text {eff }}=4500 \mathrm{~K}$, such a composite star yields a more or less constant offset of the veiling by 0.2 .

Another consequence of a reduced $T_{\text {eff }}$ in cool areas is that it will change the shape of spectral lines formed in these regions, which will influence the total spectrum if cool areas contribute significantly to the total spectrum. Fortunately, a change in line shape cannot be compensated for by adjusting the level of veiling. Therefore, if the wings of spectral lines are properly reproduced in the recovered veiled synthetic spectrum, this confirms that $T_{\text {eff }}$ is properly chosen. An excellent example of temperature-sensitive lines are the Mg lines around $5210 \AA$; at relatively low effective temperatures (below $4000 \mathrm{~K}$ ), the shapes of these lines change considerably. In our simulation with a star that is for $50 \%$ covered with cool spots, such a shape change is apparent, mostly in the wings of this line complex. However, from a comparison between the results of our calculations in this spectral region and the observed spectra, it is evident that we are able to reproduce the shape of these temperature-sensitive lines very well. The only exception is RU Lup, for which all stronger lines show some level of emission. Because we did not take into account the presence of cool spots on the stellar surface in our spectral syntheses such excellent agreement indicates that there appears to be no extended cool regions on the stellar surface of our target stars and that our estimates of the effective temperature of the photosphere are correct.

Magnetic activity may also give rise to hot regions on the stellar surface (plages). As these regions contribute more strongly to the spectrum than cool spots, these features are more likely to be visible in the spectrum than cool spots. In non-Zeeman-sensitive lines, deviations from LTE line profiles occur through changes in the temperature structure of the atmosphere, producing shallow line profiles similar to veiling. However, strong magnetic fields of several $\mathrm{kG}$ will only change the line depth with 5-10\% in Zeeman sensitive lines (Basri et al. 1990), and less in non-Zeeman-sensitive lines (Rüedi et al. 1997). Also, the effect is not the same for all lines, as within a given segment absorption lines will sample a range of Landé factors. As we do not claim a better accuracy of our veiling determinations than $10 \%$, magnetic fields are not expected to affect our veiling measurements. Still, the distribution of hot regions over the surface of the star may be inhomogeneous which would give rise to asymmetries in the observed profiles that are modulated by stellar rotation. We do not detect such deviations from our line shapes, which we take as an indication that hot regions, if present and detectable in our observations, are distributed homogeneously over the stellar surface.

\section{Conclusions}

In this paper we used spectral synthesis in order to investigate the photosphere and veiling spectrum of five CTTS. Although using spectral synthesis is untraditional in T Tauri research, it provides us with a good handle on the statistical errors and how these propagate into the final result. In order to model the photospheric contribution to the spectrum of CTTS we obtained for each star new measurements of $T_{\text {eff }}, \log g,[\mathrm{Fe} / \mathrm{H}]$, $v \sin i, v_{\text {rad }}, v_{\text {mic }}$ and $v_{\text {mac }}$. Even though we limited ourselves to 1-dimensional plane-parallel LTE calculations with solar abundances, the results are very satisfying. After correction for the veiling continuum the difference between the synthetic profiles and the observations is similar to what can be expected from the SNR of the observations. By comparing with high-quality spectra of the Sun and 61 Cyg A, we could establish that our method of measuring veiling and our atomic line lists are satisfying.

The overall shape of the recovered veiling spectra agrees very well with results in previous studies using wTTS or mainsequence dwarfs as templates for measuring veiling. These studies were performed with different telescopes and instruments, which shows that the shape of the veiling continuum is not the result of instrumental effects. The synthetic templates we calculated therefore must be similar to the observed templates used for their methods. In the strongest veiled stars we find that there exists a region around $5000-5700 \AA$ where veiling measurements are systematically higher than would be expected from purely continuous radiation. This region is most dominantly present in our observations of RU Lup, and is also present in some previous observations of strongly accreting CTTS. The interpretation of this feature is a topic for further investigation.

Acknowledgements. We would like to thank C. M. Johns-Krull for useful discussions about our veiling method and for providing the high-quality spectrum of $61 \mathrm{Cyg}$ A. The referee, G. Basri, is thanked for his valuable comments and suggestions to improve the article. We also acknowledge P.S. Barklem for correcting and commenting the article. This research has made use of the SIMBAD database, operated at CDS, Strasbourg, France.

\section{References}

Basri, G., Marcy, G. W., \& Valenti, J. A. 1990, ApJ, 360, 650

Basri, G., \& Batalha, C. 1990, ApJ, 363, 654

Bertout, C., Basri, G., \& Bouvier, J. 1988, ApJ, 330, 350

Bertout, C. 1989, ARA\&A, 27, 351

Calvet, N., \& Gullbring, E. 1998, ApJ, 509, 802

Chelli, A. 1998, A\&A, 349, L65

Gahm, G., Liseau, R., Gullbring, E., \& Hartstein, D. 1993, A\&A, 279, 477 
Ghosh, P., \& Lamb, F. K. 1979, ApJ, 234, 296

Guenther, E. W., Lehmann, H., Emerson, J. P., \& Staude, J. 1999, A\&A, 341, 768

Gustafsson, B., Edvardsson, B., Eriksson, K., et al. 2003, A Grid of Model Atmospheres for Cool Stars. In Stellar Atmosphere Modeling, ed. I. Hubeny, D. Mihalas, \& K. Werner, ASP Conf. Ser., 288

Hartigan, P., Kenyon, S. J., Hartmann, L., et al. 1991, ApJ, 382, 617

Hartigan, P., Hartmann, L., Kenyon, S., \& Hewett, R. 1989, ApJS, 70, 899

Hartmann, L., Calvet, N., Gullbring, E., \& D’Allesio, P. 1998, ApJ, 495,385

Hauschildt, P. H., Allard, F., \& Baron, E. 1999, ApJ, 512, 377

Herbig, G. H., \& Bell, K. R. 1988, Lick Obs. Bull., 1111, 1

Johns-Krull, C. M., \& Hatzes, A. P. 1997, ApJ, 487, 896

Johns-Krull, C. M., \& Valenti, J. A. 2000, in Stellar Clusters and Associations: Convection, Rotation, and Dynamos, ed. R. Pallavicini, G. Micela, \& S. Sciortino, ASP Conf. Ser., 198, 371 Joy, A. H. 1945, ApJ 102, 168

Kenyon, S. J., \& Hartmann, L. 1987, ApJ, 323, 714

Königl, A. 1991, ApJ, 370, L39
Kupka, F., Piskunov, N., Ryabchikova, T. A., Stempels, H. C., \& Weiss, W. W. 1999, A\&AS, 138, 119

Kurucz, R. L., Furenlid, I., Brault, J., \& Testerman, L. 1984, National Solar Observatory Atlas, No. 1 (Tucson: NSO)

Lamzin, S. A., Stempels, H. C., \& Piskunov, N. E. 2001, A\&A, 369, 965

Muzerolle, J., Calvet, N., \& Hartmann, L. 2001, ApJ, 550, 944

Padgett, D. L. 1996, ApJ, 471, 847

Piskunov, N. E., Kupka, F., Ryabchikova, T. A., Weiss, W. W., \& Jeffery, C. S. 1995, A\&AS, 112, 525

Piskunov, N., \& Valenti, J. 2002, A\&A, 385, 1095

Rice, J. B., \& Strassmeier, K. G. 1996, A\&A, 316, 164

Rüedi, I., Solanki, S. K., Mathys, G., \& Saar, S. H. 1997, A\&A, 318, 429

Ryabchikova, T. A., Piskunov, N. E., Stempels, H. C., Kupka, F., \& Weiss, W. W. 1999, Phys. Scr., T83, 162

Stempels, H. C., \& Piskunov, N. 2002, A\&A, 391, 595 (Paper I)

Uchida, Y., \& Shibata, K. 1985, PASJ, 37, 515

Valenti, J. A., Basri, B., \& Johns, C. M. 1993, AJ, 106, 202

Valenti, J., \& Piskunov, N. 1996, A\&AS, 118, 595 